\title{
Early and Late Mechanisms of Surround Suppression in Striate Cortex of Macaque
}

\author{
Ben S. Webb, ${ }^{2}$ Neel T. Dhruv, ${ }^{1}$ Samuel G. Solomon, ${ }^{3}$ Chris Tailby, ${ }^{1}$ and Peter Lennie ${ }^{1}$ \\ ${ }^{1}$ Center for Neural Science, New York University, New York, New York 10003, ${ }^{2}$ School of Psychology, University of Nottingham, Nottingham NG7 2RD, \\ United Kingdom, and ${ }^{3}$ Institute of Biomedical Research, The University of Sydney, New South Wales 2006, Australia
}

The response of a neuron in striate cortex to an optimally configured visual stimulus is generally reduced when the stimulus is enlarged to encroach on a suppressive region that surrounds its classical receptive field (CRF). To characterize the mechanism that gives rise to this suppression, we measured its spatiotemporal tuning, its susceptibility to contrast adaptation, and its capacity for interocular transfer. Responses to an optimally configured grating confined to the CRF were strongly suppressed by annular surrounding gratings drifting at a wide range of temporal and spatial frequencies (including spatially uniform fields) that extended from well below to well above the range that drives most cortical neurons. Suppression from gratings capable of driving cortical CRFs was profoundly reduced by contrast adaptation and showed substantial interocular transfer. Suppression from stimuli that lay outside the spatiotemporal passband of most cortical CRFs was relatively stronger when the stimulus on the CRF was of low contrast, was generally insusceptible to contrast adaptation, and showed little interocular transfer. Our findings point to the existence of two mechanisms of surround suppression: one that is prominent when high-contrast stimuli drive the CRF, is orientation selective, has relatively sharp spatiotemporal tuning, is binocularly driven, and can be substantially desensitized by adaptation; the other is relatively more prominent when low-contrast stimuli drive the $\mathrm{CRF}$, has very broad spatiotemporal tuning, is monocularly driven, and is insusceptible to adaptation. Its character suggests an origin in the input layers of primary visual cortex, or earlier.

Key words: macaque monkey; striate cortex; receptive field; surround suppression; context; cortical feedback

\section{Introduction}

In primary visual cortex (V1), the receptive fields of most neurons contain two regions with different properties: a "classical receptive field" (CRF) within which changes in contrast directly affect the discharge of the neuron, and a larger surrounding region, the "suppressive surround," within which contrast stimuli do not alone elicit a response but generally suppress the response to a stimulus on the CRF (for review, see Allman et al., 1985; Gilbert, 1992; Fitzpatrick, 2000; Wörgötter and Eysel, 2000; Albright and Stoner, 2002).

The source of suppression is unknown, although several of its properties implicate cortex: it is tuned for orientation and most potent when the spatial characteristics of the surround stimulus match those preferred by the CRF (Blakemore and Tobin, 1972; Fries et al., 1977; Nelson and Frost, 1978; DeAngelis et al., 1994; Sillito et al., 1995; Cavanaugh et al., 2002a). Moreover, in cat, a surround stimulus in one eye can suppress the response to the CRF stimulus in the other (DeAngelis et al., 1994). Suppression from outside the CRF is evident in lateral geniculate nucleus

Received Aug. 12, 2005; revised 0ct. 26, 2005; accepted Nov. 1, 2005

This work was supported by National Institutes of Health Grants EY 04440 and EY 13079. B.S.W. was partly supported by a Wellcome prize studentship, and S.G.S. was supported by Australian National Health and Medical Research Council Grant 211247. We thank Mehrdad Jazayeri, John Krauskopf, and Adam Kohn for useful discussions, Jon Peirce and Najib Majaj for help with data collection, and Anita Disney for help with histology.

Correspondence should be addressed to Peter Lennie, Center for Neural Science, New York University, 2-4 Washington Place, New York, NY 10003. E-mail: pl@cns.nyu.edu.

DOI:10.1523/JNEUROSCI.3414-05.2005

Copyright $\odot 2005$ Society for Neuroscience 0270-6474/05/2511666-10\$15.00/0
(LGN) neurons (Solomon et al., 2002; Ozeki et al., 2004; Webb et al., 2005; and previous work to which they refer), and this might be expected to find some expression in the behavior of cortical neurons. Although work in cat (Ozeki et al., 2004) does implicate subcortical mechanisms in surround suppression observed in V1, no work in primate bears on this. This is an issue of particular interest in macaque, where it seems (Krüger, 1977) that in LGN the suppressive surround mechanisms are weak or absent in parvocellular neurons.

In this study, we have tried to establish the contributions of early and late mechanisms to surround suppression in V1 by exploiting the very different visual characteristics of two groups of neurons. Those in LGN and the input layers of cortex have broad spatiotemporal tuning and are monocularly driven; therefore, suppression originating in them might be expected to carry this signature. Beyond the input layers of V1, and in extrastriate cortex, neurons generally have narrow spatiotemporal tuning and are binocularly driven, so suppression originating there should carry a distinctively different signature.

\section{Materials and Methods}

Preparation and recording. We recorded extracellularly from 348 neurons in V1 in 14 male macaque monkeys (Macaca fascicularis) anesthetized with sufentanil citrate. These recordings were part of a larger series of studies. We prepared and maintained each monkey during recording as described in Solomon et al. (2004b).

Visual stimuli and presentation. Achromatic sinusoidal gratings of the required size, orientation, spatial frequency, and contrast, and moving or 
flickering at specified rates, were displayed on a calibrated Eizo T966 monitor (Ishikawa, Japan; refresh rate, 90 or $120 \mathrm{~Hz}$; mean luminance, 50 $\mathrm{cd} / \mathrm{m}^{2}$ ), or an Iiyama (Nagano, Japan) HM 204DT monitor (refresh rate, $200 \mathrm{~Hz}$; mean luminance, $33 \mathrm{~cd} / \mathrm{m}^{2}$ ). All gratings were defined by modulation about this mean luminance. Independently adjustable mirrors were placed in front of the eyes to bring the foveas onto separate regions of the screen.

For each cell, we mapped the CRF in the dominant eye with the smallest patch of drifting grating (typically $0.1-0.2^{\circ}$ in diameter) that elicited a reliable response. Then, in the following order, we determined the orientation tuning, spatial-frequency tuning, and temporal-frequency tuning of a cell with small (typically $1-2^{\circ}$ ) patches of drifting grating. For binocular cells, we made the same measurements in the nondominant eye. For our main experiments, we generally presented two independently controllable stimuli: one within a circular aperture confined to the CRF and the other within an annular aperture overlying the suppressive surround. The diameter of the grating on the CRF was established by measuring the responses to a centered patch of less than saturating contrast, presented at a series of increasing sizes, and choosing the smallest that elicited the largest response; the CRF grating was then always presented at a contrast just below that which would evoke a saturated response. The inner diameter of the annular grating was established by measuring the responses to the annulus presented alone at a series of inner diameters (holding the outer diameter fixed) and choosing the smallest diameter that elicited no response. This diameter was never smaller than the size of the optimal grating patch on the CRF; when it was larger than the diameter of the optimal patch, we held the gap between annulus and patch at the mean luminance of the screen. The outer diameter of the annular grating was set to between $6^{\circ}$ and $8^{\circ}$.

To measure the spatial-frequency tuning of surround suppression, we presented to the CRF a grating of preferred orientation moving at the preferred spatial and temporal frequency, and in a surrounding annulus varied the spatial frequency of a grating moving at the same rate, presented at the preferred orientation of the CRF and at the orthogonal orientation. Spatial frequency ranged in equal logarithmic steps around that preferred by the CRF. The set of annular stimuli also included a spatially uniform field modulated in time with the same contrast and rate as the gratings. The series of measurements also included trials in which the CRF stimulus was presented alone and the annular stimulus was presented alone. To measure the temporal-frequency tuning of surround suppression we used the same procedure, except that the spatial frequency of the annular stimulus was held constant at the value preferred by the CRF, and its temporal frequency ranged in equal logarithmic steps from 0.6 to $30 \mathrm{~Hz}$.

To explore the binocularity of surround suppression, we delivered an optimal grating patch to the CRF of the dominant eye, alone and in the presence of an annular stimulus to the dominant eye, to the nondominant eye, or to both eyes together (the dominant eye was the one that gave the larger response to an optimal stimulus on its CRF). Because responses to binocular stimulation of the CRF often depend on the relative spatial phases of stimuli to the two eyes (Ohzawa and Freeman, 1986a; Smith et al., 1997), the surround grating was presented at a range of spatial phases.

For the above experiments, we randomized the order of stimuli within a set, which included a uniformly illuminated screen at the mean luminance. Each trial lasted $2 \mathrm{~s}$ and was repeated 10 times, with a blank screen between each presentation for $0.5 \mathrm{~s}$.

To measure the contrast sensitivity of the surround, the CRF was stimulated with an optimal grating, presented alone or together with an annular one. The contrast of the annular grating ranged in equal logarithmic steps between 0.03 and 1.0. Each stimulus set included a blank screen. Stimuli in a set appeared in random order, and each set was repeated 10 times.

To characterize the susceptibility of surround suppression to contrast adaptation, we measured its contrast sensitivity before, during, and after recovery from prolonged stimulation of the surround by a high-contrast annular grating. Before and after recovery from adaptation, each test stimulus appeared for $1 \mathrm{~s}$ with a $5.5 \mathrm{~s}$ blank between presentations. During adaptation and after an initial period of $30 \mathrm{~s}$ of adaptation to a full contrast annular grating in the surround, each test stimulus was pre- sented for $1 \mathrm{~s}$, followed by a $5 \mathrm{~s}$ "top-up" presentation of the adapting annulus, followed by a $0.5 \mathrm{~s}$ blank before the cycle was repeated.

Data analysis. We recorded the times of action potentials with a resolution of $100 \mu$ s and extracted mean discharge rate $\left(F_{0}\right)$ and the amplitude of the Fourier component at the frequency of stimulation $\left(F_{1}\right)$. If $F_{1}$ was greater than the $F_{0}$ cells were classified as simple; otherwise, they were classified as complex (Skottun et al., 1991).

To characterize the change in $F_{0}$ or $F_{1}$ caused by stimuli in the surround, we calculated a suppression index (SI) (Cavanaugh et al., 2002a) that expresses suppression as a fraction of the response to a preferred stimulus alone on the CRF (see Fig. $1 A$ ) as follows:

$$
S I=\frac{\left(R_{\text {preferred }}-R_{\text {preferred }+ \text { annulus }}\right)}{R_{\text {preferred }}},
$$

where $R$ is the difference between the spontaneous firing rate and the stimulus-elicited firing rate $\left(F_{0}\right)$ for complex cells and is $F_{1}$ for simple cells. We use the SI as a measure of the strength of the surround.

To characterize the spatial and temporal tuning of CRF and of the surround, we fitted spatial- and temporal-frequency tuning curves for the CRF and surround suppression with the following descriptive function:

$$
R=R_{\max } \exp \left(\frac{-\left(x-x_{\max }\right)^{2}}{\sigma(x)}\right),
$$

where the parameter $\sigma(x)$ can be either $\sigma_{-}$or $\sigma_{+}$, depending on whether $x<x_{\max }$ or $x>x_{\max }$, respectively. The parameters $R_{\max }, x_{\max }, \sigma_{-}$and $\sigma_{+}$ were free to vary in the fits (Freeman et al., 2002).

To characterize the contrast sensitivity of the surround, we fit the following model:

$$
R=m+R_{\max }-b \frac{c_{\text {surr }}^{\mathrm{n}}}{\left(c_{\text {surr }}^{\mathrm{n}}+\operatorname{SI} c_{50}^{\mathrm{n}}\right)},
$$

where $c_{\text {surr }}$ is the contrast of the grating in the surround. Three terms are in the model: the spontaneous activity, $m$; the maximum response to the grating on the receptive field, $R_{\max }$ and a subtractive term. The subtractive term describes the suppressive surround with a Naka-Rushton model, where $b$ and $\mathrm{SI} c_{50}$ are its response gain and contrast sensitivity, respectively (Kohn and Movshon, 2003).

To characterize the spatial phase tuning of surround suppression, we fitted measurements with a sinusoid and took the ratio of the amplitude to the mean as a phase sensitivity index (PSI) (DeAngelis et al., 1994). This takes values between 0 and 1 .

We characterized the quality of fit to the data as the percentage of response variance across stimuli explained by an equation (Carandini et al., 1997). We obtained the fits by minimizing the mean square error between the model and the data by using the fmincon function of the Optimization Toolbox for Matlab (version 6.5; Mathworks, Natick, MA). The models characterized their respective data sets well, explaining in all cases $>80 \%$ of the variance, and in most cases $>90 \%$ of the variance.

\section{Results}

Enlarging an otherwise optimal grating beyond the size of the CRF engages a suppressive mechanism that reduces the responsiveness of most V1 neurons (Fig. $1 A$ ). Figure 1, $B$ and $C$, shows for simple and complex cells, respectively, the distribution of the SI among the cells that had peak responses of at least $10 \mathrm{imp} / \mathrm{s}$. The distributions for simple and complex cells did not differ reliably. We report here only on neurons in which the suppression index was $\geq 0.2$ when measured with gratings of optimal orientation and spatial frequency (Fig. $1 B, C$, cells represented in all but the first bin). Among the cells that were admitted, simple and complex cells were not distinguished by their responses and are pooled in what follows. Receptive fields were located within $5^{\circ}$ of the fovea. 
Spatial- and temporal-frequency tuning of suppression

Figure 2 shows examples of spatialfrequency tuning curves for the CRF (top row) and surround suppression (bottom row) obtained from two complex cells and a simple cell. For each cell, measurements were made at the temporal frequency preferred by the CRF. Curves for CRF tuning are based on responses to the CRF stimulus presented alone; those for surround tuning are based on the SI, calculated as described in Materials and Methods (Eq. 1). For all three neurons, stimuli restricted to the CRF yielded responses that were sharply attenuated at low and high frequencies, as is typical of most V1 neurons (De Valois et al., 1982; Foster et al., 1985). The spatial-frequency tuning curves for surround suppression had a very different shape. With a surrounding grating of the orientation preferred by the neuron (Fig. 2 , filled circles), the suppression was strongest at low frequencies and sharply attenuated at high frequencies. Modulation of a spatially uniform field that in two neurons elicited no response from the CRF (Fig. $2 A, C)$ and barely elicited a response from the third (Fig. $2 B$ ) almost extinguished the responses of all three neurons. For each neuron, the strength of suppression at higher spatial frequencies varied with the orientation of the grating in the surround, being stronger at the orientation preferred by the CRF than at the orthogonal orientation (Fig. 2, filled squares). To characterize spatial-frequency tuning, we fit Equation 2 to the data for the CRF and for the surround (Fig. 2, smooth lines).

We were particularly interested in how the spatial-frequency tuning curve of the surround was positioned in relation to the tuning curve for the CRF. To characterize this for our population, we calculated aggregate spatial-frequency tuning curves for the CRF and for the surround. The range of spatial frequencies used to obtain an individual curve was centered on the value preferred by each neuron. We therefore normalized spatial frequency to this value before averaging. Figure $3, A$ and $B$, shows the average normalized spatial-frequency tuning curves for the CRF and the surround, respectively. The average spatial frequency preferred by the CRF was 2.98 cycles/degree; the average spatial frequency preferred by the surround was 2.2 times lower. Smooth lines show the best fitting solutions of Equation 2. Figure 3, $A$ and $B$, makes clear that the spatial-frequency tuning of the surround is very much broader than that of the CRF.

To characterize the low-frequency loss of responsiveness of the CRF and surround, Figure $3 C$ shows the SI for a spatially uniform annulus plotted against the CRF response to a spatially uniform field, normalized to the peak SI and peak CRF response, respectively. Figure $3 D$ provides a corresponding picture of the means of distributions.
B

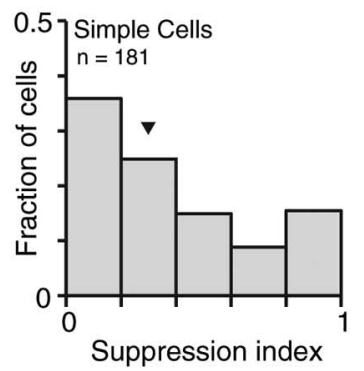

C

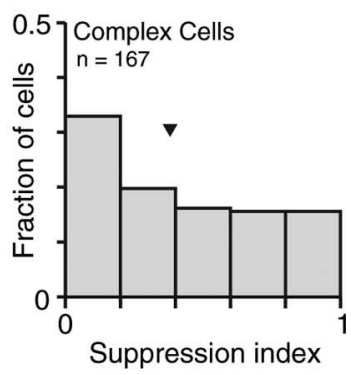

Figure 1. $\quad \boldsymbol{A}$, Size-tuning of a V1 complex cell, illustrating the method for calculating the $S I$. The patch size at which the response reached its peak is taken to be the optimal stimulus for the CRF. SI is calculated as $x / y$. The cell had no maintained discharge. $\boldsymbol{B}, \boldsymbol{C}$,
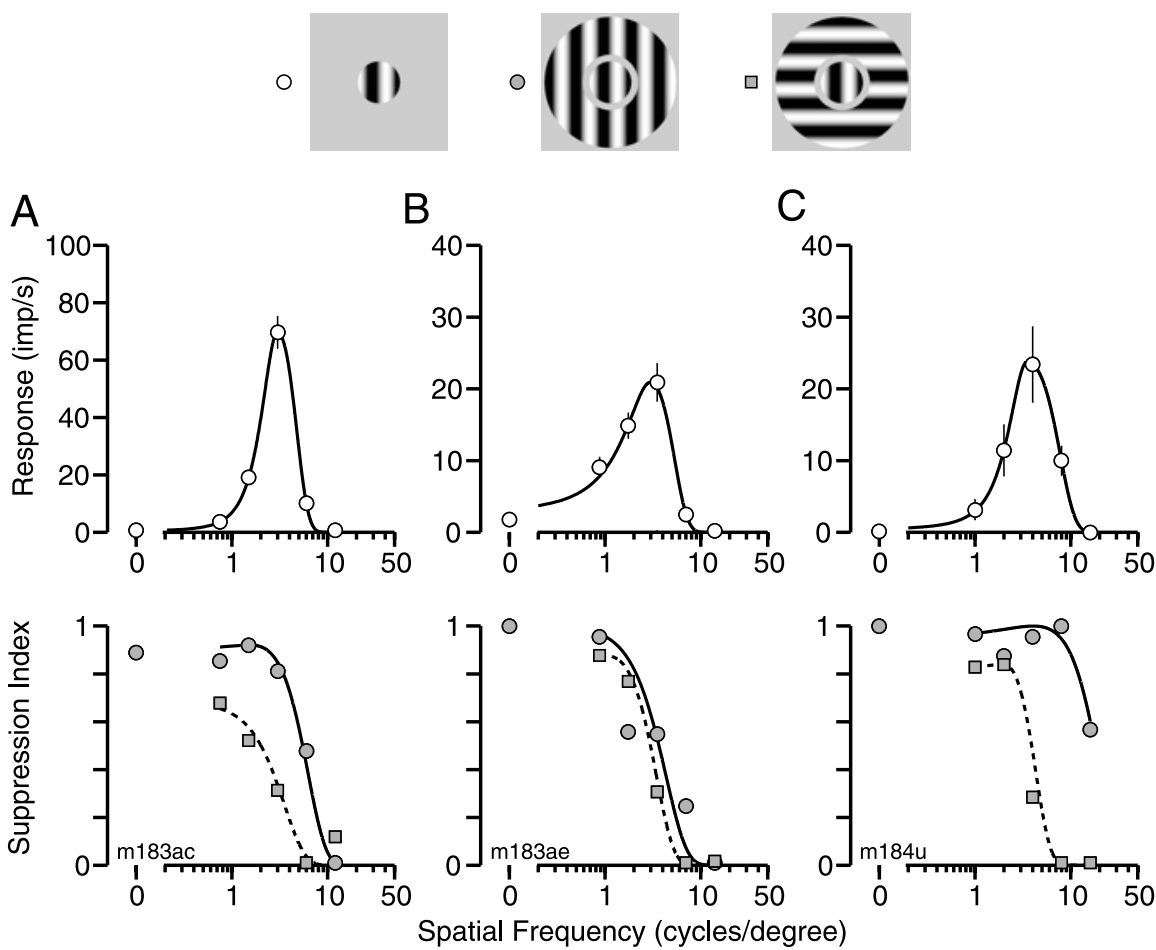

Figure 2. Spatial-frequency tuning curves for the CRF and the surround. $\boldsymbol{A}-\boldsymbol{C}$, For three different cells, the tuning of the CRF (top row) and of the surround (bottom row) are shown. In this and subsequent figures, icons show the essentials of the stimulus configuration. The gap between the circular patch and the annulus is for clarity of illustration only and did not necessarily exist; the orientation preferred by the CRF is always identified by a vertical grating, and the orthogonal orientation is always identified by CRF (circles) and one at the orthogonal orientation (squares). Smooth lines show best-fitting solutions to Equation 2. Surround grating contrast: $1.0 ;$ CRF grating contrast: $\boldsymbol{A}, 0.4 ; \boldsymbol{B}, 0.5 ; \boldsymbol{C}, 0.8$. Temporal frequency: $\boldsymbol{A}, 10 \mathrm{~Hz} ; \boldsymbol{B}, 6 \mathrm{~Hz} ; \boldsymbol{C}, 5 \mathrm{~Hz}$.

high-frequency loss of responsiveness and shows the SI to the spatial frequency at five times the peak against the CRF response at five times the peak normalized to the peak SI and peak CRF response, respectively. We used this method of characterizing high-frequency loss because it was not possible to compute a conventional measure of bandwidth for the surround. In Figure 3, $C$ and $D$, all but a few points lie above the unity line, making clear that in most neurons the surround responds to both low and high spatial frequencies that generally do not drive the CRF.

We characterized the temporal-frequency tuning of the CRF and of the surround with methods analogous to those used to characterize spatial-frequency tuning. All gratings had the spatial frequency preferred by the CRF and (for the patch on the CRF) 


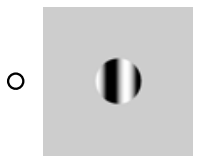

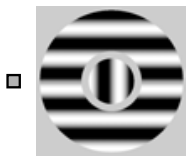

A

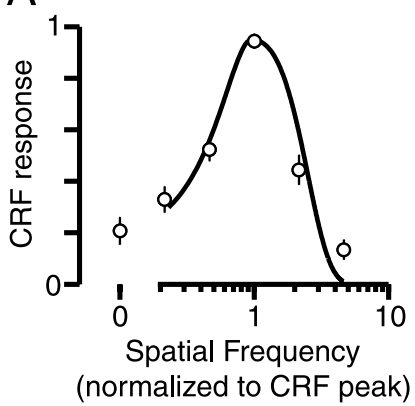

B

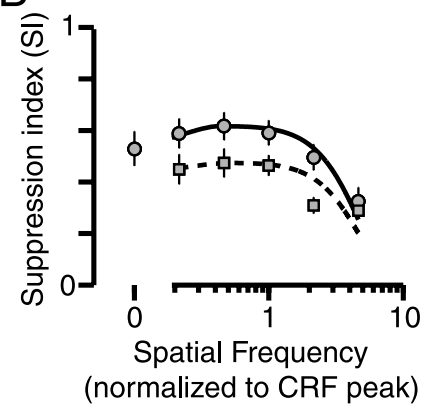

C

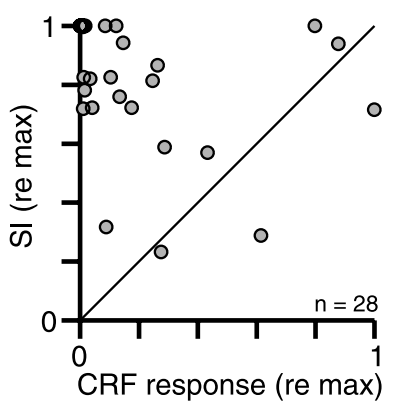

D

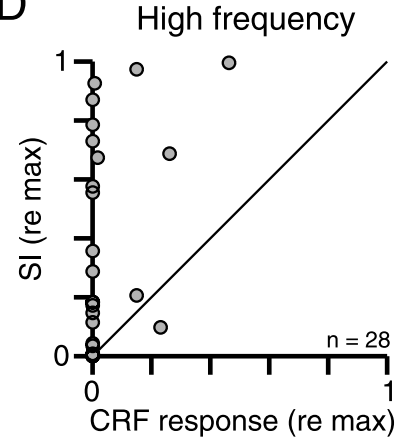

Figure 3. Average spatial frequency tuning curve for the CRF $(\boldsymbol{A})$ and the surround $(\boldsymbol{B})$. Spatial frequency was normalized to the value preferred by the CRF. In $A$, the response of each cell was normalized to its peak value before being averaged. In $\boldsymbol{B}$, filled circles show surround tuning for a grating at the orientation preferred by the CRF; filled squares show tuning for a grating at the orthogonal orientation. C, Comparison of normalized SI and CRF responses to spatially uniform fields. Responses in each case were normalized to the maximum obtained. $\boldsymbol{D}$, Comparison of normalized SI and normalized CRF response for the spatial frequency at five times that preferred by the CRF. max, Maximum value of SI (ordinate) or maximum CRF response (abscissa).

the preferred temporal frequency. Figure 4 shows temporalfrequency tuning curves obtained from two complex cells and a simple cell, for the CRF stimulated alone (top row) and for surround suppression (bottom row). The CRFs were sharply tuned for temporal frequency, as is characteristic of cortical neurons (Hawken et al., 1996). The surrounds were very broadly tuned: both low and high temporal frequencies that barely elicited a response from the CRF produced potent surround suppression. Indeed, for two neurons (Fig. $4 A, C$ ), surround suppression was almost uniformly strong over the whole range of temporal frequencies studied.

Figure 5, $A$ and $B$, shows average normalized temporalfrequency tuning curves for the CRF and the surround, respectively. The average temporal frequency preferred by the CRF was $4.99 \mathrm{~Hz}$; the average temporal frequency preferred by the surround was $9.23 \mathrm{~Hz}$. Temporal-frequency tuning of the surround is essentially flat over the range of frequencies that we studied, and at both low and high frequencies it extends well beyond the passband of the CRF. To characterize the low-frequency loss of responsiveness of the CRF and surround, Figure $5 C$ shows the SI for $0.5 \mathrm{~Hz}$ plotted against the CRF response to $0.5 \mathrm{~Hz}$, normalized to the peak SI and peak CRF response, respectively. Figure $5 D$ provides a corresponding picture of the high-frequency loss of responsiveness and shows the $\mathrm{SI}$ at $30 \mathrm{~Hz}$ against the CRF response at $30 \mathrm{~Hz}$ normalized to the peak SI and peak CRF response, respectively. (The surround responded so well at high temporal frequencies that it was not possible to provide a conventional characterization of its high-frequency roll-off.) In Figure 5, $C$ and $D$, the points lie well above the unity line, making clear that in most neurons the surround responds well to both low and high temporal frequencies that do not drive the CRF.

Gratings of spatial frequencies well below those to which most V1 neurons responded elicited powerful surround suppression. Gratings drifting at temporal frequencies well below and well above those to which most V1 neurons responded also elicited powerful suppression. These observations implicate mechanisms early in the visual pathway, before the development of sharp spatiotemporal tuning. Were these in retina or LGN, or even in the input layers of V1, the suppression would be indifferent to the orientation of the grating on the surround. We know that is not wholly the case (Figs. 3, 5) (Levitt and Lund, 1997; Cavanaugh et al., 2002a; Jones et al., 2002), but if early mechanisms are substantially involved, suppression should be least selective for orientation when spatial and temporal frequencies are farthest from those preferred by cortical neurons. Figures $3 B$ and $5 B$ show that, for gratings of the preferred spatial and temporal frequency, suppression is slightly greater for gratings at the preferred than the orthogonal orientation [the average SI drops from 0.59 to 0.46 (Fig. $3 B$ ) and from 0.76 to 0.54 (Fig. $5 B$ )], and that at higher spatial frequencies, or temporal frequencies $>20 \mathrm{~Hz}$, suppression is indifferent to the orientation of the grating.

\section{Contrast sensitivity of broadly tuned suppression}

To characterize the contrast sensitivity of the mechanisms with broad spatial tuning, we stimulated the CRF with an optimal grating while varying the contrast of an annular stimulus presented to the surround. The annulus contained a grating of the optimal spatial frequency at the preferred orientation or the orthogonal orientation, or it contained a spatially uniform field. Figure 6, $A$ and $B$, shows how the responses of two complex cells varied with the contrast of the stimulus falling on the surround. Open circles indicate responses in the presence of an annular grating of the same spatial frequency and orientation as that falling on the CRF; filled circles indicate responses in the presence of a grating of the same spatial frequency but orthogonal orientation; shaded circles indicate responses in the presence of a spatially uniform field.

Smooth curves drawn through the points are solutions to Equation 3, obtained by fitting all three sets of points simultaneously and constrained so that only the sensitivity parameter $\left(\mathrm{SI} c_{50}\right)$ can vary. This constraint has the benefit of providing a single index of sensitivity ( $\mathrm{SI}_{50}$ ) and captures the data well; allowing $R_{\max }$ also to vary increased the variance explained from 87 to $89 \%$ over all neurons on which we made measurements. Using SI $c_{50}$ as the measure of sensitivity, the surround was 10.5 times (Fig. 6A) and 6.5 times (Fig. 6B) less sensitive to an orthogonal grating than to an optimal one and approximately 3.5 times (Fig. $6 A, B)$ less sensitive to a spatially uniform field than to an optimal grating.

Figure 7 shows how surround sensitivity to different stimuli is distributed over the population of cells in which we measured it. Figure $7 A$ shows SI $c_{50}$ for suppression evoked by the preferred annular grating against $\mathrm{SI} c_{50}$ for suppression evoked by the orthogonal grating. In most neurons, the surround was much less sensitive to the orthogonal grating (median SI $c_{50}, 1.15 \pm 0.99$ ) than to the preferred one (median SI $c_{50}, 0.18 \pm 0.09$ ). Figure $7 B$ 
shows $\mathrm{SI} c_{50}$ for suppression evoked by the preferred annular grating against $\mathrm{SI} c_{50}$ for suppression evoked by a spatially uniform field. On average, the surround was $\sim 2.5$ times less sensitive to a uniform field (median SI $c_{50}, 0.44 \pm 0.77$ ) than to an optimal grating. The corresponding ratio for the CRF, estimated from spatial-frequency tuning curves for the same cells (data not shown), shows that the average response to a spatially uniform field was $>10$ times less than the response to a preferred grating.

Levitt and Lund (1997) showed that surround suppression became less selective for orientation when the CRF was driven by low-contrast stimuli. This might reflect a relatively stronger influence at low contrast of mechanisms with broad spatiotemporal tuning. We explored this in 11 neurons by measuring contrast sensitivity to surround stimuli at two levels of CRF contrast. The connected pairs of points in Figure 7 show the values of SI $c_{50}$ obtained when the CRF was driven by gratings of lower contrast (open circles) and higher contrast (filled circles). In Figure $7 B$, almost all of the open circles lie substantially to the left of their filled counterparts, indicating that the broadly tuned suppressive mechanisms are relatively more sensitive at lower CRF contrasts.

The relatively high sensitivity of the surround to spatially uniform fields strongly implicates mechanisms in the input layers of V1, or earlier, yet the substantial orientation selectivity for gratings paradoxically implicates mechanisms beyond the input layers of V1. To gain a better understanding of the site at which these putative mechanisms act, we examined the susceptibility of the surround to contrast adaptation.

\section{Independent mechanisms revealed by contrast adaptation}

Prolonged exposure to a high-contrast pattern brings about a temporary loss of sensitivity in V1 neurons (Movshon and Lennie, 1979; Ohzawa et al., 1985; Sclar et al., 1989). This "contrast adaptation" is also found in M-cells in LGN (Solomon et al., 2004c) but is not pronounced at low temporal frequencies that strongly adapt cortical neurons. Cavanaugh et al. (2002a) showed that in a V1 receptive field the surround and CRF are independently adaptable. We exploited this to learn whether the presumptive early and late components of surround suppression could be distinguished by their susceptibility to adaptation.

We recorded responses of V1 neurons to optimal gratings of fixed contrast on the CRF, in the presence of annular gratings or uniform fields at a range of contrasts. We made sets of measurements before, during, and after exposure to adapting stimuli in the surround (the spatial configuration of the adaptor was systematically varied; for details, see Materials and Methods). Figure 8 shows, for three neurons, how adaptation affects the suppressive capacity of the surround. In Figure $8, A$ and $B$, filled circles show the average of responses obtained before adaptation and after recovery, when the surround stimuli were gratings of the preferred spatial frequency and orientation; open circles show responses when the surround was adapted to the preferred grat-
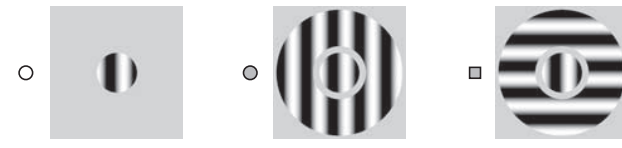

C

B
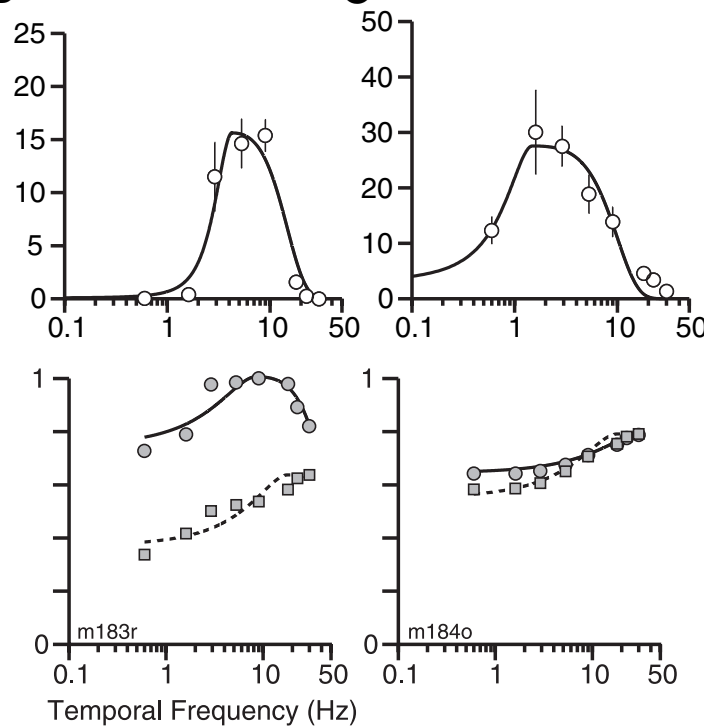

Temporal Frequency $(\mathrm{Hz})$

Figure 4. Temporal-frequency tuning curves for the CRF and surround. $\boldsymbol{A}-\boldsymbol{C}$, For three neurons, the tuning of the CRF (top row) best-fitting solutions to Equation 2. Surround grating contrast: 1.0 . CRF grating contrast: $A, 0.8 ; B, 0.5 ; C, 0.8$. Spatial frequency: $\boldsymbol{A}, 0.4$ cycles/degree; $\boldsymbol{B}, 1.2$ cycles/degree; $\boldsymbol{C}, 1.4$ cycles/degree.

ing. Points at the left margin show the responses to the CRF stimulus presented alone, and the shaded squares show the responses to the adapter alone. The smooth lines are the best-fitting solutions to Equation 3, obtained by allowing only $R_{\max }$ and SI $c_{50}$ to vary between the unadapted and adapted states. The fits for these and other cells were well constrained and were only slightly less good when only SI $c_{50}$ was allowed to vary. Because the change brought about by adaptation was reflected principally in $\mathrm{SI} c_{50}$, our subsequent analysis focused on that.

In the unadapted state, suppression evoked by a preferred grating on the surround was evident at low contrasts, reaching half its maximum value below $10 \%$ contrast (Fig. 8A,B) (unadapted $\left.\operatorname{SI} c_{50}, 0.06,0.09\right)$. Adaptation to the preferred grating in the surround greatly reduced the capacity of the surround to suppress responses (Fig. $8 A, B$ ) (adapted $\mathrm{SI}_{50}, 0.17,0.78$ ). An annular adapting grating at the orientation orthogonal to that preferred by the CRF also reduced the sensitivity of the surround to a preferred grating (Fig. $8 \mathrm{~B}$, open triangles) (adapted SI $c_{50}$, $0.57)$, but not to the same degree as an adapter of the preferred orientation. In some neurons (Fig. $8 \mathrm{~B}$ ), an adapting grating falling on the surround slightly reduced the peak response to a CRF stimulus alone, presumably through direct adaptation of the CRF (Fig. $8 A-C$ ) (unadapted $R_{\text {max }}, 58.4,38.3$, and $87.3 \mathrm{imp} / \mathrm{s}$; adapted $R_{\max }, 56.6,27.6$, and $83.3 \mathrm{imp} / \mathrm{s}$ ). This was always a small effect and observed in fewer than half the neurons that we studied; were it to have influenced our estimate of the change in surround sensitivity, it would have lead us to an "underestimate."

We also examined the effects of adapting the surround with spatially uniform fields (i.e., stimuli that evoke substantial surround suppression but are ineffective when delivered to the CRF) but found no conditions under which this reduced the sensitivity of the surround. Figure $8 \mathrm{~A}$ shows that adaptation to a uniform 

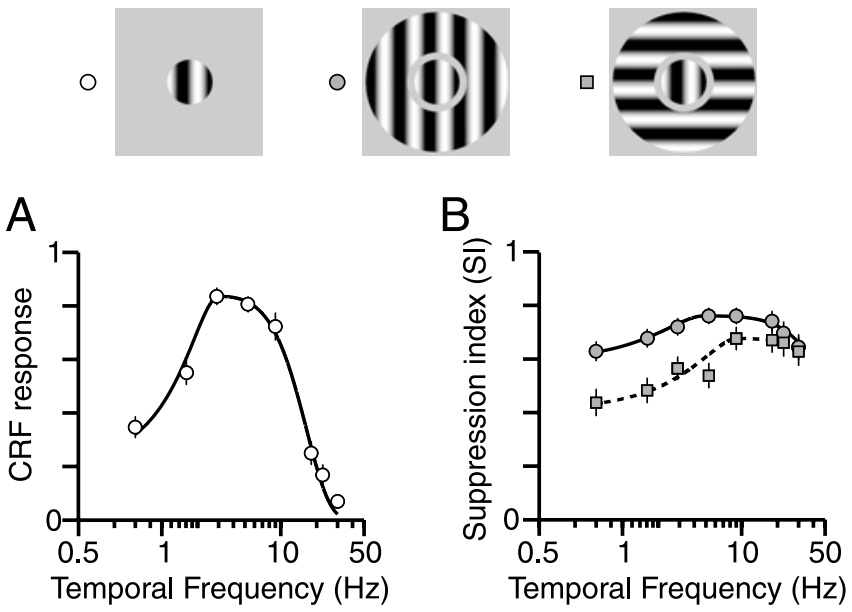

C $0.5 \mathrm{~Hz}$ drift

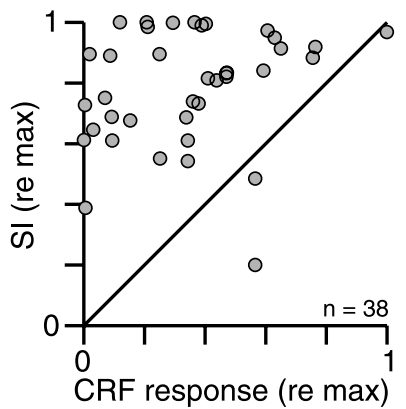

D

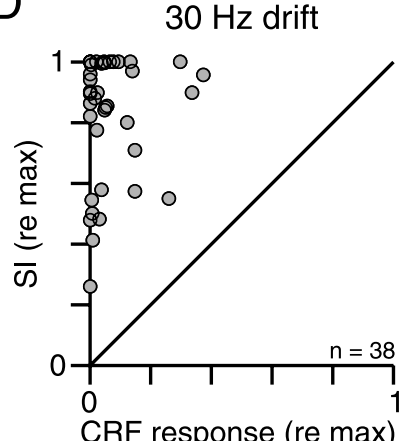

Figure 5. Average temporal frequency tuning curve for the $\operatorname{CRF}(\boldsymbol{A})$ and the surround $(\boldsymbol{B})$. In $\boldsymbol{A}$, the response of each cell was normalized to its peak value before being averaged. Continuous lines show best-fitting solutions to Equation 2 . In $\boldsymbol{B}$, filled circles show surround tuning for a grating at the orientation preferred by the CRF; filled squares show tuning for a grating at the orthogonal orientation. C, Comparison of SI for $0.5 \mathrm{~Hz}$ and CRF response to the same frequency. Responses in each case were normalized to the maximum obtained. $\boldsymbol{D}$, Comparison of normalized SI for the highest temporal frequency and normalized CRF response to the highest temporal frequency. max, Maximum value of $\mathrm{SI}$ (ordinate) or maximum CRF response (abscissa).
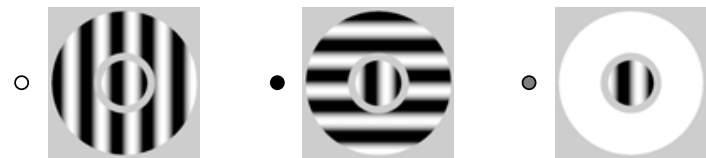

A

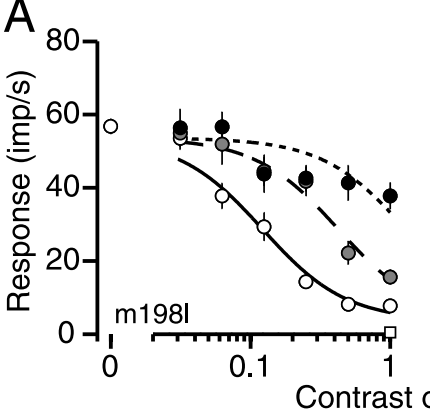

B

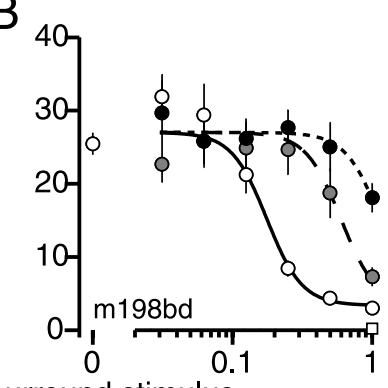

Figure 6. Contrast sensitivity of surround suppression. Responses of two neurons to optimal gratings presented to the CRF, as a function of the contrast of annular stimuli containing the preferred grating (open circles), the orthogonal grating of the same spatial frequency (filled circles), or a spatially uniform field (shaded circles). (RF grating contrast: $\boldsymbol{A}, 0.4 ; \boldsymbol{B}, 0.5$. Parameter values for curves: $R_{\mathrm{max}}: \boldsymbol{A}, 58.27 ; \boldsymbol{B}, 27.36 ; \mathrm{Sl}_{50}: \boldsymbol{A}, 0.12,0.42,1.29 ; \boldsymbol{B}, 0.18,0.56,1.15$; for a preferred grating surround, a uniform field surround, and an orthogonal grating surround, respectively.
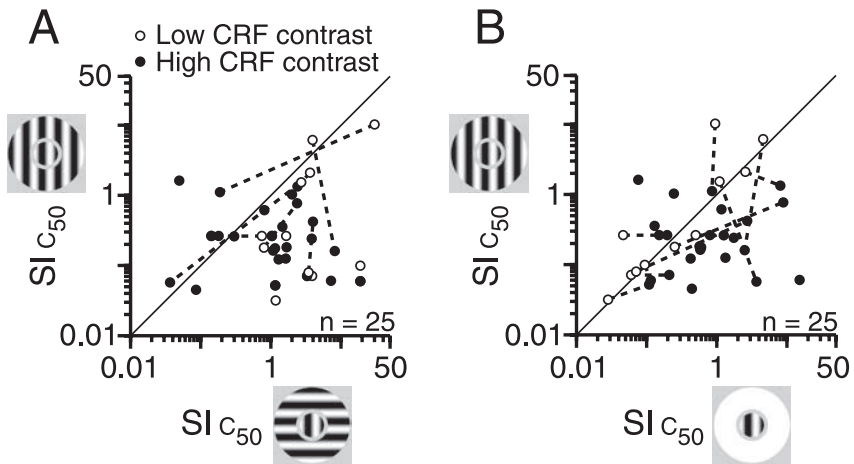

Figure 7. Contrast sensitivity of surround suppression is regulated by CRF contrast. $A, \mathrm{SIC}_{50}$ for suppression by an optimal annular grating versus $\mathrm{Sl}_{50}$ for suppression by an annular grating at the orthogonal orientation. Open circles show values obtained with a low contrast grating on the CRF; filled circles show values obtained with a high (but not saturating) contrast grating on the CRF. Dashed lines connect points from the same cell. $\boldsymbol{B}, \mathrm{SI}_{50}$ for suppression by an optimal annular grating versus $\mathrm{SI}_{50}$ for suppression by a spatially uniform annulus. Other conventions as in $\boldsymbol{A}$.

annulus flickering at $50 \mathrm{~Hz}$ (a stimulus that itself evoked strong surround suppression; shaded diamond) had no effect on the suppression evoked by the optimal grating (open squares; adapted $\left.S I c_{50}, 0.07\right)$. Figure $8 C$ shows that adaptation to a spatially uniform annulus flickering at the optimal temporal frequency had no effect on the sensitivity of the surround to a spatially uniform annulus flickering at the same frequency. In this and all neurons that we examined, suppression brought about by a uniform annulus (Fig. $8 C$, filled squares) (unadapted $\mathrm{SI}_{50}$, 0.51 ) was unaffected or slightly sensitized by adaptation to uniform annuli (Fig. $8 C$, open squares) (adapted SI $c_{50}, 0.40$ ). Suppression by uniform annuli was similarly unaffected by adaptation to drifting gratings (Fig. $8 C$, open circles) (adapted $\mathrm{SI}_{50}$, $0.49)$.

Figure 9 summarizes, from best-fitting solutions to Equation 3 , the changes in surround $\mathrm{SI}_{50}$ brought about by adapting the surround. Figure $9 A$ shows the changes in $S I c_{50}$ brought about by adaptation to an annular grating of the orientation preferred by the CRF. In almost all cells, adaptation substantially increased the contrast needed to suppress responses (geometric mean SI $c_{50}$ : unadapted 0.13; adapted 0.57; $t=-3.12 ; p<0.005$ ). Adaptation to an annular grating orthogonal to that preferred by the CRF (Fig. 9B) also increased the contrast needed for the preferred grating to suppress responses of most neurons ( $\mathrm{SI}_{50}$ : unadapted, 0.25 ; adapted, 0.67 ). Adaptation to a spatially uniform annulus flickering at the preferred temporal frequency (Fig. 9 C, filled circles) and high temporal frequency (Fig. $9 C$, open squares) had no effect on the sensitivity of the surround to a preferred grating ( $\mathrm{SI}_{50}$ : unadapted, 0.14 ; adapted, 0.16$)$ or a spatially uniform annulus (data not shown).

The loss of surround sensitivity brought about by adaptation to an orthogonal grating, a fourfold increase in $\mathrm{SI}_{50}$ (Fig. $9 \mathrm{~B}$ ), is unlikely to reflect adaptation in LGN. On the one hand, adaptation to the grating brings about a greater loss than would be expected from changes in the sensitivity of M-neurons (Solomon et al., 2004c); on the other hand, adaptation to uniform fields (potent stimuli in LGN) brings about no loss at all.

All of this suggests the existence of two distinct mechanisms of surround suppression: one early, broadly tuned, prominent when the CRF is driven by low-contrast stimuli, and relatively immune to adaptation; the other later, narrowly tuned, prominent when the CRF is driven by high-contrast stimuli, and sus- 
ceptible to adaptation. Early mechanisms are more likely to be monocularly driven, so we examined this by characterizing interocular transfer of suppression.

\section{Interocular transfer of suppression}

Binocular interactions are pronounced only beyond the input layers of V1 (Hubel and Wiesel, 1968). We can exploit this to help pinpoint the origin of the suppressive signal. If it originates in the visual pathway before the combination of signals from the two eyes, a surround stimulus presented to one eye should not suppress the response to a CRF stimulus in the other. DeAngelis et al. (1994) examined this in cat and found that in almost all cells a surround stimulus in one eye suppressed the response to a CRF stimulus in the other, but not as strongly as when both stimuli were presented to the same eye. Binocular interactions have not been examined in cat with stimuli other than those to which cortical neurons are well tuned; they have not been studied at all in monkey.

We examined binocular interactions in neurons that met our criterion for surround suppression in the dominant eye and also met two additional criteria for binocularity: they responded reliably $(>10 \mathrm{imp} / \mathrm{s})$ to a grating delivered to the CRF in the nondominant eye, and their responses to a CRF stimulus in the dominant eye were suppressed to $<90 \%$ of peak by an annular grating in the nondominant eye.

Binocular interactions can depend substantially on the relative phases of stimuli to the two eyes (Ohzawa and Freeman, 1986a; Smith et al., 1997); a sensitivity to phase would establish that signals are summed linearly up to the point of binocular combination (Ohzawa and Freeman, 1986b). We characterized the sensitivity of 12 cells to the spatial phase of the annular grating in each eye alone and their sensitivity to the relative spatial phases of annular gratings presented to both eyes together. We fitted sinusoids to the three sets of data from each neuron, and from each fit we calculated a spatial PSI (see Materials and Methods). The PSI is 1 if suppression is fully modulated and 0 if suppression is unmodulated. In most neurons, suppression was essentially indifferent to the spatial phase of the grating on the surround in the dominant eye alone (median PSI, $0.13 \pm 0.05$ ), the nondominant eye alone (PSI, $0.24 \pm 0.07$ ), and both eyes together (PSI, $0.17 \pm 0.06)$. This agrees with the observations of DeAngelis et al. (1994) in cat.

We characterized interocular transfer of suppression in 15 cells. Figure $10 \mathrm{~A}$ compares the SI (Eq. 1) when a preferred annular grating was delivered to the nondominant eye versus the dominant eye. When the CRF of one eye was clearly dominant, a surround grating delivered to that eye always suppressed the response more than did a surround grating delivered to the nondominant eye. This can be expressed as a transfer ratio: nondominant eye SI/dominant eye SI. For the neurons represented in Figure $10 \mathrm{~A}$, the median transfer ratio was $0.77 \pm 0.05$. The interocular transfer makes clear that some component of suppression acts after the combination of signals from the two eyes and must originate in cortex. For the measurements shown in Figure $10 \mathrm{~A}$, the spatiotemporal properties of the surround stimuli were those preferred by the CRF and therefore would maximize the contribution of cortical mechanisms to the suppressive signal. Because the broad spatiotemporal bandwidth of suppression points to some role for early mechanisms, which are less likely to be driven binocularly, we also explored the transfer of suppression when the surround was driven by stimuli that fell outside the spatial or temporal passbands of most cortical neurons.

Figure 10, $B$ and $C$, compares the relative efficacy of interocular suppression brought about by an optimal grating with that brought about by a spatially uniform field flickering at the preferred temporal frequency (Fig. $10 \mathrm{~B}$ ) and a spatially uniform field flickering at $50 \mathrm{~Hz}$ (Fig. 10C). For most neurons, there was considerably less transfer of suppression for a uniform annulus flickering at the preferred temporal frequency (median transfer ratio, $0.14 \pm 0.09$ ) or a high temporal frequency (median transfer ratio, $0.51 \pm 0.13$ ) than for a preferred grating. This behavior would be expected from a surround signal that arises in two component mechanisms: a monocular one with broad spatiotemporal tuning and a binocular one with narrower spatiotemporal tuning.

\section{Discussion}

We have shown that the spatiotemporal tuning of surround suppression is always much broader than that of the CRF and extends well outside the range that drives most cortical neurons (De Valois et al., 1982; Hawken et al., 1996). In the low spatial-frequency domain, our results agree with those of DeAngelis et al. (1994) in cat, who found the surround to have low-pass tuning. In the temporal frequency domain, our results differ from those in cat. Durand et al. (2002), found that the sensitivity of the surround to high temporal frequencies did not extend beyond those that drive cortical neurons. Species differences might be important here, but beyond that, Durand et al. (2002) would have had less opportunity to discover the broad bandwidth because they worked with a substantially narrower range of temporal frequencies than we did. We found that the suppression was relatively indifferent to the orientation of the grating on the surround, particularly when the surround was driven by high temporal frequencies and when the CRF was stimulated by gratings of low contrast. The latter 

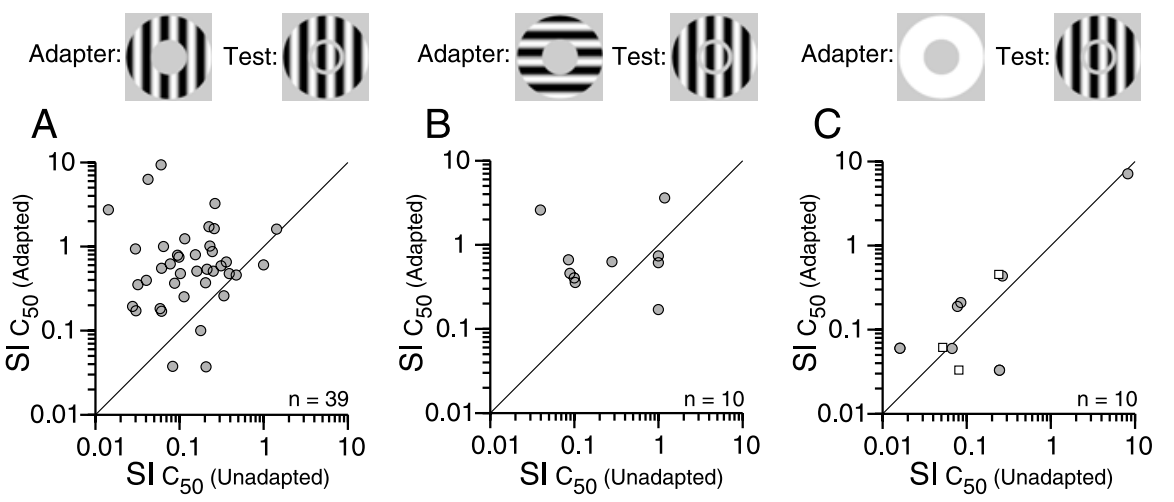

Figure 9. Change brought about by adaptation in the sensitivity $\left(\mathrm{Sl}_{\mathrm{C}_{50}}\right)$ of the surround to a grating of the preferred orientation and spatial frequency. $\boldsymbol{A}$, Change caused by adaptation to an annular grating of the preferred orientation. $\boldsymbol{B}$, Change caused by adaptation to an annular grating of the orthogonal orientation. $\boldsymbol{C}$, Change caused by adaptation to a spatially uniform annulus. Circles show the effects of adaptation to an annulus flickering at the temporal frequency preferred by the CRF; squares show the effects of adaptation to an annulus flickering at $50 \mathrm{~Hz}$.
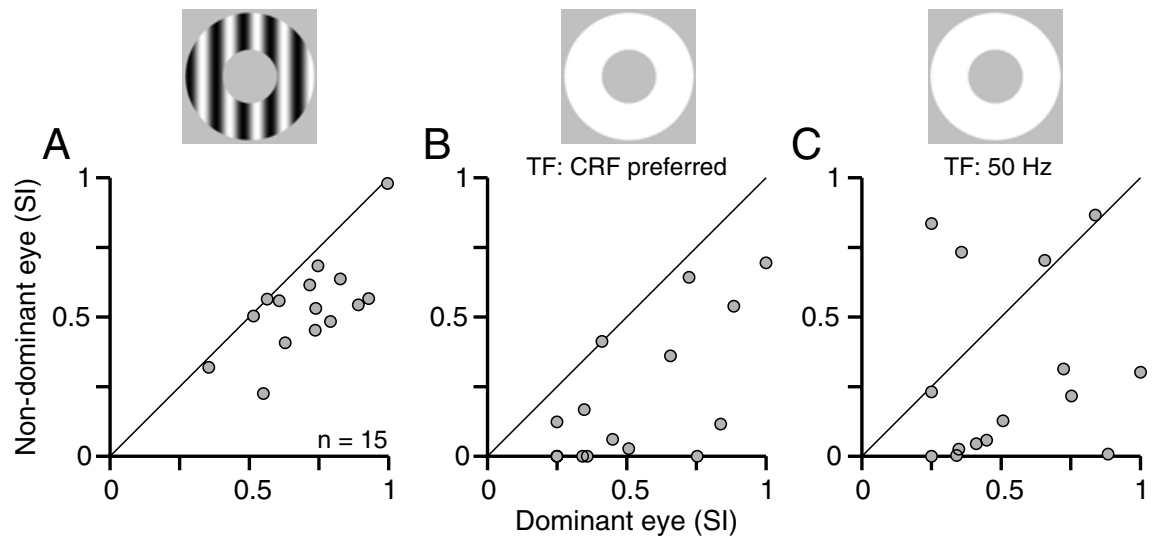

Figure 10. Interocular transfer of surround suppression. $\boldsymbol{A}$, Comparison of suppression brought about by a preferred annular grating in the surround of the nondominant eye versus that brought about by a preferred annular grating in the surround of the dominant eye. $\boldsymbol{B}$, Same as $\boldsymbol{A}$, except showing suppression brought about by an annular spatially uniform field flickering at the preferred temporal frequency. $\boldsymbol{C}$, Same as $\boldsymbol{A}$, except showing suppression brought about by an annular spatially uniform field flickering at $50 \mathrm{~Hz}$. TF, Temporal frequency.

observation is in agreement with Levitt and Lund (1997) and Cavanaugh et al. (2002a) who found that when the CRF was driven by gratings of below-saturating contrast (as we used), suppression was much more broadly tuned for orientation than when saturating contrasts were used.

The surround was substantially desensitized by contrast adaptation when the spatial and temporal frequencies of the adapting grating fell within the passbands of most cortical neurons but was negligibly desensitized by adaptation to very low spatial frequencies and high temporal frequencies that generally fail to drive cortical neurons but do themselves bring about strong surround suppression. We found substantial interocular transfer of the suppressive signal when the spatial and temporal frequencies of the surround grating were those optimal for the CRF [in agreement with observations by DeAngelis et al. (1994) in cat], but much less transfer when the spatial and temporal frequencies were outside the ranges that excite most cortical neurons (Fig. $10)$.

We were initially concerned that some of the sensitivity of the surround to spatially uniform fields might be an artifact of our use of an annular stimulus with a sharp inner edge, which provides energy at higher spatial frequencies. We explored this through simulation but could find no circumstance under which an edge artifact produced suppression as strong as that brought about by the spatially uniform annulus. Had there been a consequential artifact, the annulus would have acted like a low-contrast grating, but our results show that it did not. Changing the contrast of the stimulus on the CRF had different effects on the suppressive capacities of annular gratings and uniform fields: a uniform annulus that elicited strong suppression did not adapt the surround, but an annular grating did; a grating that adapted the surround (and reduced the suppression evoked by a grating) had no effect on the suppressive capacity of a spatially uniform annulus.

\section{Early and late suppressive mechanisms}

A simple account that accommodates all of our observations conceives the surround as originating in two distinct components. One that is most evident when the CRF is driven by high-contrast stimuli has relatively sharp spatiotemporal tuning, is binocularly driven, and can be substantially desensitized by adaptation; the other, prominent at lower CRF contrasts (Fig. 7) (Levitt and Lund, 1997), is spatiotemporally broad band, monocularly driven, and relatively insusceptible to adaptation. These narrowly and broadly tuned processes can be conceived as late and early, respectively.

The narrowly tuned component must originate in cortex, certainly beyond the input layers in $\mathrm{V} 1$ and possibly outside $\mathrm{V} 1$ altogether (Angelucci et al., 2002; Cavanaugh et al., 2002a; Levitt and Lund, 2002). It is orientation selective and, equally important, can be direction selective (Levitt and Lund, 1997; Cavanaugh et al., 2002b). Its influence transfers binocularly, and it is substantially desensitized by contrast adaptation.

The broadly tuned component of suppression lacks the signature of most cortical neurons. Few cells in V1 respond at all to the high temporal frequencies that elicited strong suppression (Hawken et al., 1996), and few respond to modulation of spatially uniform fields (De Valois et al., 1982; Foster et al., 1985). The broadly tuned suppression does have the spatiotemporal signature of neurons in LGN and of neurons in the input layers of striate cortex (Blasdel and Fitzpatrick, 1984; Hawken et al., 1996). An early locus is also implied by the suppression being monocularly driven and is consistent with the observation (Bair et al., 2003; Müller et al., 2003) that some components of suppression are expressed as fast as the excitatory drive to the CRF. Surround suppression is already evident in LGN neurons (Solomon et al., 2002; Webb et al., 2005). In marmoset, this is as strong, on average, as that observed in cortical neurons (Fig. $1 B, C$ ) (Cavanaugh et al., 2002a) but is distributed differently: more V1 neurons show strong suppression and more show no suppression. In macaque retina (Solomon et al., 2004a) and LGN (our unpublished observations), surround suppression is hard to discern in P-cells. We therefore think it likely that a substantial component of the 


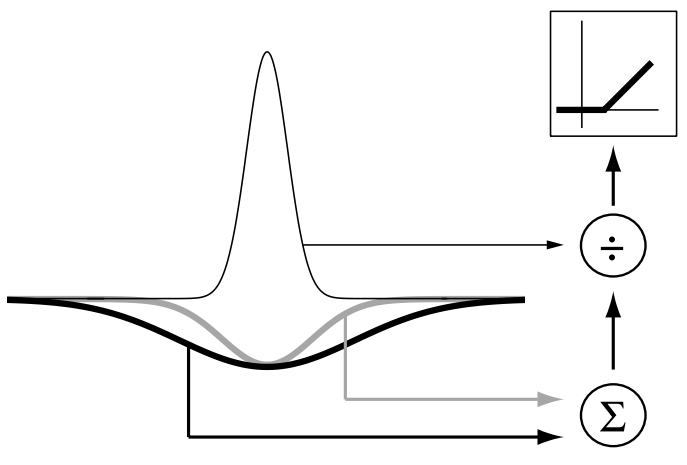

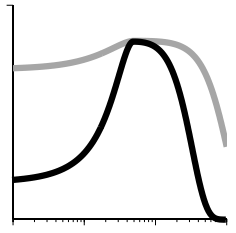

Spatial Frequency

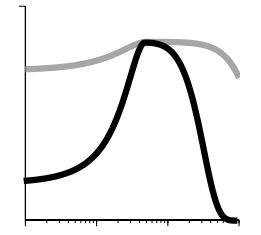

Temporal Frequency

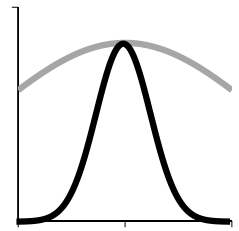

Orientation
Figure 11. Hypothetical organization and spatial distribution of sensitivity in the early suppressive mechanism (shaded lines) and the late mechanism (solid lines). The early mechanism is broadly tuned in orientation and in spatial and temporal frequency and is smaller; the late mechanism is more sharply tuned for orientation and for spatial and temporal frequency and is larger. Signals from the two mechanisms act divisively on the signal from the CRF.

broadly tuned suppression arises in the input layers of V1. Hirsch et al. (2003) described inhibitory interneurons in cat layer 4 that seemed well equipped to provide broadly tuned suppression. Although none have yet been characterized in monkey cortex, it seems likely that similar neurons exist there.

The sum of our observations can be accommodated by an extension of the model developed by Cavanaugh et al. (2002a), in which the receptive field is conceived as a Gaussian-weighted central mechanism (the CRF), the response of which is divided by the signal from a larger, concentric, Gaussian-weighted surround. We suppose the surround to consist of two components with a summed signal that divides the response of the CRF. The early (broadly tuned) component accumulates signals from a smaller region, or one more heavily weighted toward the center, than the later (narrowly tuned) one. As a result, the broadly tuned mechanism is relatively more sensitive to the stimulus falling on the CRF. The upshot is that when the contrast on the CRF is low, a spatially uniform annulus is a relatively more effective suppressor (Fig. 7), and, overall, the surround is less selective for orientation (Levitt and Lund, 1997) than when the contrast on the CRF is high.

The early component presumably contributes to the suppression from orthogonal annular gratings but cannot be the sole source of it, because surround suppression evoked by an optimal grating can be adapted by an orthogonal grating but not by spatially uniform annuli that are nevertheless often more effective suppressors (Figs. 8, 9). This finding implicates a late mechanism and points to it being constructed from a pool of cells that are narrowly tuned for spatial frequency and broadly tuned for orientation (or are narrowly tuned for both spatial frequency and orientation but have preferred orientations broadly distributed in the pool). Figure 11 shows schematically how we conceive the two components of the surround and its relationship to the CRF.

\section{Relationship to other gain controls}

Surround suppression has generally been considered a distinct form of gain control unrelated to the mechanisms of cross- orientation inhibition and contrast gain control (Bonds, 1989; Geisler and Albrecht, 1992; Heeger, 1992; Sengpiel et al., 1998), that regulate sensitivity within the CRF (cf. Freeman et al., 2002). The contrast gain control is not orientation selective and is weakly binocular. Two mechanisms seem to be involved, at least in cat. One can be driven dichoptically, is strongest at low temporal frequencies, and is susceptible to contrast adaptation ( $\mathrm{Li}$ et al., 2005; Sengpiel and Vorobyov, 2005). The other can be driven only monocularly, is particularly strongly expressed at high temporal frequencies (Allison et al., 2001; Freeman et al., 2002; Li et al., 2005), and is not susceptible to contrast adaptation (Freeman et al., 2002; Li et al., 2005). The characteristics of this mechanism are much like those of the broadly tuned component of surround suppression, a similarity that encourages the idea that they constitute a single mechanism that extends through and beyond the receptive field.

Were a single mechanism responsible for both phenomena, then its properties would become tightly constrained. First, the action of the contrast gain control is firmly established as divisive, but it has been harder to establish that the surround acts in the same way (Sceniak et al., 2001; Cavanaugh et al., 2002a; Müller et al., 2003). To the extent that the two phenomena arise in the same mechanism, the action of the surround is more firmly established as divisive (Carandini et al., 1997). Second, a recent account of the contrast gain control attributes it to the depression of the geniculocortical synapse that provides the excitatory drive of the neuron (Carandini et al., 2002; Freeman et al., 2002). For synaptic depression to explain the broadly tuned surround suppression, the surround pathways would need to be the same as those that carried the excitatory drive of the neuron. This would imply that broadly tuned suppression is accumulated over a region no larger than the CRF (the extent of which substantially exceeds that of the optimal stimulus) (Cavanaugh et al., 2002a). Some recent observations from Xing et al. (2005) bear on this possibility. In experiments quite different from ours, they characterized the dynamics of two inhibitory mechanisms that shape orientation selectivity in V1: an orientation-selective one that covers the CRF and extends well beyond it, and one that is not selective for orientation and is confined to a region about the size of the CRF. Were the broadly tuned suppressive mechanism to arise in early inhibitory interneurons (Troyer et al., 1998), it would not be constrained to the size of the CRF.

\section{References}

Albright TD, Stoner GR (2002) Contextual influences on visual processing. Annu Rev Neurosci 25:339-379.

Allison JD, Smith KR, Bonds AB (2001) Temporal-frequency tuning of cross-orientation suppression in the cat striate cortex. Vis Neurosci 18:941-948.

Allman J, Miezen F, McGuinness E (1985) Stimulus specific responses from beyond the classical receptive field: neurophysiological mechanisms for local-global comparisons in visual neurons. Annu Rev Neurosci 8:407-430.

Angelucci A, Levitt JB, Lund JS (2002) Anatomical origins of the classical receptive field and modulatory surround field of single neurons in macaque visual cortical area V1. Prog Brain Res 136:373-388.

Bair W, Cavanaugh JR, Movshon JA (2003) Time course and time-distance relationships for surround suppression in macaque V1 neurons. J Neurosci 23:7690-7701.

Blakemore C, Tobin EA (1972) Lateral inhibition between orientation detectors in the cat's visual cortex. Exp Brain Res 15:439-440.

Blasdel GG, Fitzpatrick D (1984) Physiological organization of layer 4 in macaque striate cortex. J Neurosci 4:880-895.

Bonds AB (1989) Role of inhibition in the specification of orientationselectivity of cells in the cat striate cortex. Vis Neurosci 2:41-55.

Carandini M, Heeger DJ, Movshon JA (1997) Linearity and normalization 
in simple cells of the macaque primary visual cortex. J Neurosci 17:8621-8644.

Carandini M, Heeger DJ, Senn W (2002) A synaptic explanation of suppression in visual cortex. J Neurosci 22:10053-10065.

Cavanaugh JR, Bair W, Movshon JA (2002a) Nature and interaction of signals from the receptive field center and surround in macaque V1 neurons. J Neurophysiol 88:2530-2546.

Cavanaugh JR, Bair W, Movshon JA (2002b) Selectivity and spatial distribution of signals from the receptive field surround in macaque V1 neurons. J Neurophysiol 88:2547-2556.

DeAngelis GC, Freeman RD, Ohzawa I (1994) Length and width tuning of neurons in the cat's primary visual cortex. J Neurophysiol 71:347-374.

De Valois RL, Albrecht DG, Thorell LG (1982) Spatial frequency selectivity of cells in macaque visual cortex. Vision Res 22:545-560.

Durand S, Mante V, Freeman TCB, Carandini M (2002) Temporal properties of surround suppression in primary visual cortex. In: Federation of European Neuroscience Societies Forum, p A051.056. Paris.

Fitzpatrick D (2000) Seeing beyond the receptive field in primary visual cortex. Curr Opin Neurobiol 10:438-443.

Foster KH, Gaska JP, Nagler M, Pollen DA (1985) Spatial and temporal frequency selectivity of neurones in visual cortical areas V1 and V2 of the macaque monkey. J Physiol (Lond) 365:331-363.

Freeman TC, Durand S, Kiper DC, Carandini M (2002) Suppression without inhibition in visual cortex. Neuron 35:759-771.

Fries W, Albus K, Creutzfeldt OD (1977) Effects of interacting visual patterns on single cell responses in cat's striate cortex. Vision Res 17:1001-1008.

Geisler WS, Albrecht DG (1992) Cortical neurons: isolation of contrast gain control. Vision Res 32:1409-1410.

Gilbert CD (1992) Horizontal integration and cortical dynamics. Neuron 9:1-13.

Hawken MJ, Shapley RM, Grosof DH (1996) Temporal-frequency selectivity in monkey visual cortex. Vis Neurosci 13:477-492.

Heeger DJ (1992) Normalization of cell responses in cat striate cortex. Vis Neurosci 9:181-197.

Hirsch JA, Martinez LM, Pillai C, Alonso JM, Wang Q, Sommer FT (2003) Functionally distinct inhibitory neurons at the first stage of visual cortical processing. Nat Neurosci 6:1300-1308.

Hubel DH, Wiesel TN (1968) Receptive fields and functional architecture of monkey striate cortex. J Physiol (Lond) 195:215-243.

Jones HE, Wang W, Sillito AM (2002) Spatial organization and magnitude of orientation contrast interactions in primate V1. J Neurophysiol 88:2796-2808.

Kohn A, Movshon JA (2003) Neuronal adaptation to visual motion in area MT of the macaque. Neuron 39:681-691.

Krüger J (1977) The shift-effect in the lateral geniculate body of the rhesus monkey. Exp Brain Res 29:387-392.

Levitt JB, Lund JS (1997) Contrast dependence of contextual effects in primate visual cortex. Nature 387:73-76.

Levitt JB, Lund JS (2002) The spatial extent over which neurons in macaque striate cortex pool visual signals. Vis Neurosci 19:439-452.

Li B, Peterson MR, Thompson JK, Duong T, Freeman RD (2005) Crossorientation suppression: monoptic and dichoptic mechanisms are different. J Neurophysiol 94:1645-1650.

Movshon JA, Lennie P (1979) Pattern-selective adaptation in visual cortical neurones. Nature 278:850-852.
Müller JR, Metha AB, Krauskopf J, Lennie P (2003) Local signals from beyond the receptive fields of striate cortical neurons. J Neurophysiol 90:822-831.

Nelson JI, Frost BJ (1978) Orientation-selective inhibition from beyond the classical receptive field. Brain Res 139:359-365.

Ohzawa I, Freeman RD (1986a) The binocular organization of simple cells in the cat's visual cortex. J Neurophysiol 56:221-242.

Ohzawa I, Freeman RD (1986b) The binocular organization of complex cells in the cat's visual cortex. J Neurophysiol 56:243-259.

Ohzawa I, Sclar G, Freeman RD (1985) Contrast gain control in the cat's visual system. J Neurophysiol 54:651-667.

Ozeki H, Sadakane O, Akasaki T, Naito T, Shimegi S, Sato H (2004) Relationship between excitation and inhibition underlying size tuning and contextual response modulation in the cat primary visual cortex. J Neurosci 24:1428-1438.

Sceniak MP, Hawken MJ, Shapley R (2001) Visual spatial characterization of macaque V1 neurons. J Neurophysiol 85:1873-1887.

Sclar G, Lennie P, DePriest DD (1989) Contrast adaptation in striate cortex of macaque. Vision Res 29:747-755.

Sengpiel F, Vorobyov V (2005) Intracortical origins of interocular suppression in the visual cortex. J Neurosci 25:6394-6400.

Sengpiel F, Baddeley RJ, Freeman TCB, Harrad R, Blakemore C (1998) Different mechanisms underlie three inhibitory phenomena in cat area 17. Vision Res 38:2067-2080.

Sillito AM, Grieve KL, Jones HE, Cudeiro J, Davis J (1995) Visual cortical mechanisms detecting focal orientation discontinuities. Nature 378:492-496.

Skottun BC, De Valois RS, Grosof DH, Movshon JA, Albrecht DG, Bonds AB (1991) Classifying simple and complex cells on the basis of response modulation. Vision Res 31:1079-1086.

Smith III EL, Chino YM, Ni J, Ridder III WH, Crawford ML (1997) Binocular spatial phase tuning characteristics of neurons in the macaque striate cortex. J Neurophysiol 78:351-365.

Solomon SG, White AJ, Martin PR (2002) Extraclassical receptive field properties of parvocellular, magnocellular, and koniocellular cells in the primate lateral geniculate nucleus. J Neurosci 22:338-349.

Solomon SG, Sun H, Lee BB (2004a) Surround suppression in magnocellular-pathway ganglion cells of the macaque retina. J Vision 4:782a.

Solomon SG, Peirce JW, Lennie P (2004b) The impact of suppressive surrounds on chromatic properties of cortical neurons. J Neurosci 24:148-160.

Solomon SG, Peirce JW, Dhruv NT, Lennie P (2004c) Profound contrast adaptation early in the visual pathway. Neuron 42:155-162.

Troyer TW, Krukowski AE, Priebe NJ, Miller KD (1998) Contrast-invariant orientation tuning in cat visual cortex: thalamocortical input tuning and correlation-based intracortical connectivity. J Neurosci 18:5908-5927.

Webb BS, Tinsley CJ, Vincent CJ, Derrington AM (2005) Spatial distribution of suppressive signals outside the classical receptive-field in lateral geniculate nucleus. J Neurophysiol 94:1789-1797.

Wörgötter F, Eysel UT (2000) Context, state and the receptive fields of striatal cortex cells. Trends Neurosci 23:497-503.

Xing D, Shapley RM, Hawken MJ, Ringach DL (2005) The effect of stimulus size on the dynamics of orientation selectivity in macaque V1. J Neurophysiol 94:799-812. 